\title{
A Systemic Review and Meta-Analysis of Randomized Controlled Trials of the Impact of Sleep Duration on Adiposity and Components of Energy Balance
}

\author{
Patrice L. Capers ${ }^{1,2,{ }^{*}, \text { Aaron D. Fobian }}{ }^{2,3,{ }^{*}, \text { Kathryn A. Kaiser }}{ }^{1,2,{ }^{*}, \text { Rohit Borah }}{ }^{1,4}$, and David \\ B.Allison ${ }^{1,2}$ \\ ${ }^{1}$ Office of Energetics, School of Public Health, University of Alabama at Birmingham \\ ${ }^{2}$ Nutrition Obesity Research Center, University of Alabama at Birmingham \\ ${ }^{3}$ Department of Psychiatry and Behavioral Neurobiology, University of Alabama at Birmingham \\ ${ }^{4}$ Graduate Program, School of Public Health, University of Alabama at Birmingham
}

\section{Abstract}

Background-Recent epidemiological and ecological trends in humans indicate a possible causal relationship between sleep duration and energy balance. We aimed to find experimental evidence that has tested this relationship between sleep duration and measures of body composition, food intake or biomarkers related to food intake.

Methods-We conducted a systematic literature review using six databases through August 7 , 2014. We sought reports of randomized controlled trials where sleep duration was manipulated and measured outcomes were body weight or other body composition metrics, food intake, and/or biomarkers related to eating.

Results-We found 18 unique studies meeting all criteria: eight studies with an outcome of body weight (4 - increased sleep, 4 - reduced sleep); four studies on food intake; four studies of sleep restriction on total energy expenditure and three of respiratory quotient; four studies on leptin and/or ghrelin.

Conclusions-Few controlled experimental studies have addressed the question of the effect of sleep on body weight/composition and eating. The available experimental literature suggests that sleep restriction increases food intake and total energy expenditure with inconsistent effects on integrated energy balance as operationalized by weight change. Future controlled trials that examine the impact of increased sleep on body weight/energy balance factors are warranted.

\footnotetext{
Contact Author: David B. Allison, The University of Alabama at Birmingham, 1720 2nd Avenue South, RPHB140J, Birmingham, AL 35294 -0022, dallison@uab.edu.

The first three authors contributed equally to this work

The funders had no role in study design, data collection and analysis, decision to publish, or preparation of the manuscript.

Conflicts of Interest: The authors report no conflict of interest.

Ethical Approval: Not required.
} 


\section{Keywords}

sleep; obesity; weight; total energy expenditure

\section{Introduction}

Since 1980, worldwide obesity has doubled, with $39 \%$ of adults being reported as overweight or obese in 2014. In 2013, 42 million children under the age of 5 were overweight or obese according to the World Health Organization ${ }^{1}$. In the last 30 years, obesity rates have doubled for adults and tripled among children and adolescents in the United States ${ }^{2}$. Overweight and obesity have been found to be associated with increased insulin, low-grade inflammation and leptin; they are also associated with increased fasting glucose and decreased ghrelin ${ }^{3,4}$. In many observational studies, there are reports of an inverse association between sleep duration and body weight. Over the same time period that overweight and obesity rates have increased, sleep duration in adults and adolescents has reportedly decreased $5,6,7$. This has led to the hypothesis that there may be causal links between the two phenomena.

Factors that may be related to sleep duration and obesity include socioeconomic status, culture, mental health, family dynamics ${ }^{8,9}$, use of media ${ }^{10}$, and work schedules ${ }^{11}$, among others. These factors may have oscillating influences on an individual's sleep and collectively overtime lead to a either a net sleep deficit or surplus that shifts other biological functions. While each of these factors are related to health status, it is difficult to objectively measure and control all of these factors in an experimental setting in humans. However, the co-occurrence of increased obesity and decreased sleep duration has prompted more recent research exploring the possible relationship.

Matricciani et al. reviewed trends in sleep duration data for 5-18 year olds from 20 countries and found a 75 minute average decline in sleep duration between 1905 and 2008 across most ages, sexes and regions of the world ${ }^{6}$. Large epidemiological studies have found a "doseresponse relationship between short sleep duration, excess body weight and metabolic disturbance across all age groups and in several ethnic groups." ${ }^{12}$ Studies of this nature have found short sleep duration and obesity to be significantly associated ${ }^{13}$; however, the causal pathway between these is still unclear. Some factors associated with body composition have also been found to be associated with short sleep duration, including increased insulin, inflammation, leptin and body mass index (BMI), as well as decreased blood glucose and ghrelin $^{14-17}$.

Additionally, an acute bout of short sleep has been found to be associated with alterations in food intake amounts or preferences ${ }^{18}$. From such short-term observations, it is logical to posit that changes in sleep duration may affect changes in long-term energy balance ${ }^{16,19}$. In the literature, the phrase 'energy balance' is used in more than one way. When used to refer to an outcome variable, it is often take to mean net change in body energy stores (i.e., total energy in minus total energy out per unit of time). Others use the term "positive energy balance" to indicate overweight or obese status. Except in periods of rapid short-term weight 
loss or extensive exercise, change in body weight will be nearly linearly related to change in body energy stores, and so we consider it an indicator of energy balance herein.

Researchers have begun using more targeted experimental and epidemiological methods to examine the mechanisms by which sleep duration and obesity may be related. Some sleep duration interventions involve the manipulation of sleep via time in bed (e.g. extension or restriction), behavioral strategies (e.g. cognitive based therapy), anatomical/physiological modification (e.g. continuous positive airway pressure - CPAP, tonsillectomy) or pharmaceutical interventions (e.g. prescription sleep aids). Beebe and colleagues have found that five nights of sleep restriction in adolescents may cause increased consumption of sweets, thereby potentially increasing the risk tor obesity ${ }^{20,21}$. A recent study examined habitual sleep duration, BMI, and self-reported macronutrient intake in several cohorts of people to determine whether CLOCK gene variants modified the relationship. However, many of these studies utilize self-report of food intake or food preference, which is of questionable validity. Therefore, we sought to examine only experimental studies using methods to directly observe or verify food intake.

Previous systematic reviews and meta-analyses have explored the sleep duration/obesity relationship in cohort/cross sectional ${ }^{22-24}$, longitudinal ${ }^{25}$, crossover and some randomized controlled trials (RCT) in children, adolescents, and adults. However, because of the large differences in the samples sizes and populations examined, as well as the variety of sleep durations and follow-up, it is difficult to draw clear conclusions from these prior works about whether sleep duration has causal effects on obesity-related factors. The purpose of our study was to review published randomized controlled trials to evaluate whether the available experimental evidence supports the presence of a significant relationship between sleep duration and objectively measured obesity-related factors such as body weight/body composition, energy expenditure, leptin, ghrelin, , or food intake and to obtain a quantitative estimate of associated risk from decreased sleep duration or benefit from increased sleep duration.

\section{Methods}

\section{Systematic Review of the Literatur}

searched the following databases for available articles and dissertations through August 7, 2014: Cochrane, SCOPUS, CINAHL, PubMed and Psyclnfo. We also obtained additional sources from the references of papers found in the initial search or colleagues. This review was registered with the PROSPERO registry: CRD42013006133, which can be viewed at http://www.crd.york.ac.uk/PROSPERO.

\section{Inclusion Criteria}

1. Randomized Control Trials

2. Humans

3. English language articles 
4. Independent variables: interventions designed to achieve such alterations in sleep duration. Differences in randomly assigned sleep durations (which could be increases or decreases from baseline levels) and/or behavioral

5. An outcome must be at least one of the following: weight, BMI, BMI percentile (for children), weight change, total body fat, waist circumference, food intake, physical activity, metabolic rate, or appetite-related hormones (ghrelin, glucagon, leptin, insulin). Values must be measured (not self-reported).

6. Published on or before the search date of August 7, 2014.

7. Intervention or experimental manipulation period of at least 24 hours.

\section{Exclusion Criteria}

We excluded studies with combined interventions where the unique effects of sleep duration on energy balance could not be separately evaluated. We also excluded studies of participants with serious illnesses other than obesity (e.g. metabolic syndrome, diabetes, cardiovascular disease; see registry noted above for complete list), inpatient studies on persons with anxiety and/or depression; use of medications that effect body weight; bariatric surgery; organ transplant patients; persons with mobility impairment; current drug or alcohol dependence; surgical correction to increase sleep or reduce snoring; oral appliances for airway modification; use of other airway devices for sleep apnea (e.g. CPAP); and hormone therapy.

\section{Study Search Terms}

An illustration of the search syntax used in PubMed is as follows: "Sleep" [Mesh] OR sleep*OR sleep apnea OR "sleep apnea syndromes"[Mesh] OR sleep quality OR "sleep quality” AND ((random*) OR (randomize*) OR (randomis*) OR (randomi*ed trial) OR (randomi*ed controlled trial)) AND "Exercise"[Mesh] OR "Physical Exertion"[Mesh] OR exercis* OR exertion OR physical fitness OR physical activity OR athletic performance OR physical endurance OR baseball OR basketball OR bicycling OR boxing OR football OR golf OR gymnastics OR hockey OR martial arts OR tai chi OR mountaineering OR racket sports OR tennis OR running OR jogging OR skating OR snow sports OR skiing OR soccer OR swimming OR diving OR track \& field OR volleyball OR walking OR weight lifting OR wrestling OR “sports"[Mesh] OR "Energy Metabolism”[Mesh] OR energy expenditure [ti] OR "metabolic equivalent" [Mesh] OR energy expenditure OR energy expenditure* OR metabolic equivalent*OR resting energy expenditure OR total energy expenditure OR metabolism OR energetics OR energy restriction OR basal metabolic OR bioenergetics OR resting metabolic OR indirect calorimetry OR (obes*) OR (adipos*) OR (overweight*) OR (over weight*) OR (body weight) OR (obesity) OR (obese) OR (fat adiposity) OR (overeat*) OR (over eat*) OR (overfeed*) OR (over feed*) OR "food intake" OR eating OR appetite regulation OR ingestion OR calories OR "dietary restriction" OR "food intake regulation" OR (weight gain*) OR (weight cycling) OR (weight reduc*) OR (weight loss) OR (weight losing) OR (weight maint*) OR (weight decreas*) UK (weight watch*) UK (weight diet*) UK (weight control*) UK (body mass index) OR (BMI) OR (waist 
circumference) OR (body size) OR (body composition) OR (body fat distribution) OR (skinfold thickness) OR (body weight changes) OR (body mass) OR (fat mass).

Filters applied were: Humans; English language; Randomized Controlled Trials.

\section{Study Selection}

See the PRISMA diagram in Figure 1 for the search and study selection process. Studies were screened by removing duplicates, non-English language and animal studies. Following this, a series of keywords were entered to remove articles that met our exclusion criteria. Articles were then evaluated based on title and abstract to evaluate if the studies met the inclusion/exclusion criteria before full articles were retrieved. All articles considered for full review were evaluated by the first three authors (PLC, ADF, and KAK). The most common reasons for exclusion were that the studies were not truly RCTs or body composition or energy balance factors were not reported.

\section{Data Extraction Process}

When we reached consensus on the final inclusion set of studies, we used a standard data extraction form to collect all required information for descriptive and statistical analysis. Data extraction was completed by at least two authors (KAK, PLC, ADF, RB) independently and reviewed by KAK and $\mathrm{KB}$ to resolve any data discrepancies. If there was additional information needed that was not in the published paper, an email was sent to request additional information from the contact author, followed by either a phone call or additional email two weeks later. If no response was received, the study was removed from the final analysis due to lack of complete information.

We extracted information for the following variables:

1. Number randomized

2. Participant demographics (mean age, percent female, health status, baseline weight/ BMI)

3. Intervent on detail $\mathrm{s}$

4. Protocol duration

5. Randomization method

6. Study Design

7. Mean (SD) weight change

8. Mean (SD) total sleep time change

9. Mean (SD) food intake change

10. Mean (SD) hormone(s) change

11. Method for missing data handling 


\section{Statistical Analysis}

We used Review Manager ${ }^{26}$ and Microsoft ${ }^{\circledR}$ Excel to calculate the standardized mean differences between treatment and control groups when sample sizes, means and SEs/SDs were reported, or if exact $p$ values were given between groups for change values within groups. These data were used to generate the forest plots for each comparison using Review Manager, version 5.3.5 26 .

\section{Risk of Bias Assessment}

At least two authors (PLC, ADF, KAK, RB) independently reviewed each paper and evaluated the areas of potential risk of bias according to the Cochrane Handbook guidelines ${ }^{27}$ Discrepancies in ratings were discussed until consensus was reached. The risk of bias summary figures were generated with the Review Manager software, version 5.3.5 26 .

\section{Results}

\section{Results of Literature Search}

Our initial aim was to find studies with an intervention duration of at least four weeks; however, since we found only two ${ }^{28,29}$, we expanded the duration criteria to interventions of at least 24 hours. All studies meeting criteria were of adult samples ${ }^{18,28-41}$ except two ${ }^{42,43}$. Some studies had more than one paper reporting different outcomes from the same trial. Table 1 (RCTs reporting weight outcomes) and Table 2 (RCTs and other designs with other outcomes related to energy balance) contain the selected papers with key descriptive data for each. We found a total of four unique studies that examined the effects of either increased sleep or behavioral/breath training to improve sleep duration ${ }^{28-30,42}$ where changes in body weight were reported or available upon request. We round tour studies mat examined the ettects of sleep restriction on body weight ${ }^{31,35,36,41}$. Four other studies examined effects of sleep deprivation on food purchase ${ }^{32}$, food portion size ${ }^{33}$, or food intake ${ }^{18,41}$. Several studies reported sleep related outcomes for the hormones leptin and/or ghrelin after sleep restriction ${ }^{31,38-40}$ or sleep increase ${ }^{42}$. Finally, four papers reported total energy expenditure (TEE) $)^{34,35,37,43}$ and three reported respiratory quotient (RQ) ${ }^{34,37,43}$ after sleep restriction.

\section{Results of Body Weight and Energy Expenditure Effects}

The forest plots below are key comparisons from available data that group studies by type of intervention and outcome data based on our stated variables of interest. Studies aimed at increasing sleep duration revealed inconsistent but overall significant effects on body weight, $\mathrm{p}=0.02$, with low heterogeneity $\mathrm{I}^{2}=35 \%$ (Figure 2). Studies that restricted sleep indicate some weight gain or less weight loss compared to control in three out of the four studies, but no significant effect overall ( $p=0.08$, Figure 3 ). The latter group also reflects a high degree of heterogeneity $\left(\mathrm{I}^{2}=81 \%\right)$. Four studies that restricted or disturbed sleep indicate a non-significant increase in TEE overall $(\mathrm{p}=0.11$, Figure $4 \mathrm{~A})$ with high heterogeneity, $\mathrm{I}^{2}=87 \%$. When examining the effect of sleep manipulation on RQ the results were inconclusive among the three studies $(\mathrm{p}=0.86$, Figure $4 \mathrm{~B})$. The limited number and 
small sample sizes of available studies reporting TEE and RQ limit conclusions of effects on these outcomes.

\section{Results of Leptin and Ghrelin Effects}

See Figures 5A and 5B for overall comparisons. One study in adults demonstrated no significant effects on leptin or ghrelin in adults after 8 nights of two-thirds of normal sleep time compared to 8 nights of normal sleep ${ }^{31}$. A study of children found no significant change in fasting leptin levels nor in ghrelin when sleep was decreased by 1.5 hours as compared to 1.5 hours increase in sleep ${ }^{42}$. Overall amongst the four studies available for leptin outcomes, there was no significant effect $(\mathrm{p}=0.84)$ with high heterogeneity, $\mathrm{I}^{2}=$ $75 \%$. Overall amongst the four studies available for ghrelin outcomes, there was no significant effect $(\mathrm{p}=0.83)$ with high heterogeneity, $\mathrm{I}^{2}=77 \%$. Of these reporting ghrelin, one showed a significant effect of sleep restriction lowering ghrelin on average ${ }^{39}$, while another ${ }^{40}$ demonstrated a significant increase when sleep was restricted to four hours versus being extended to 10 hours. A non-randomized crossover study ( 8 hours sleep for two nights or 4 hours sleep for two nights - see Table 2) comparing fasting levels to post-prandial levels of ghrelin and leptin demonstrated no significant effects on these outcomes ${ }^{44}$.

\section{Food Intake Outcomes}

Several studies assessed the effects of sleep restriction on food intake. However, due to the inconsistency in measuring food intake (direct observation versus self-report, variety of foods made available) and the primary use of laboratory settings instead of real-world settings, we opted not to meta-analyze these studies. We will instead narratively summarize the results here. Both Brondel et al. ${ }^{18}$ (four hours' sleep for one night) and O'Keeffe/StOnge et al. ${ }^{36,45}$ (four hours sleep for five nights) reported that reduced sleep significantly increased ad libitum food intake in a laboratory setting. Additionally, Hogenkamp et al. reported that adults chose larger portion sizes and reported feeling hungrier after one night of total sleep deprivation as compared to a night of normal sleep ${ }^{33}$. Another study demonstrated that men purchased significantly greater calories after a night of total sleep deprivation. Eight nights of two-thirds of normal sleep time resulted in a significant increase in energy intake ${ }^{31,35}$, and four consecutive nights of four hours sleep in a laboratory setting resulted in a significant increase in energy intake compared to the control group ${ }^{41}$. Finally, Hart et al. demonstrated a significant increase in food intake in children (self-reported 24 hour dietary recalls) after a decrease of 1.5 hours from normal sleep (sleep diaries verified by actigraphy) as compared to an increase of 1.5 hours from normal sleep time ${ }^{42}$. Overall, these studies suggest that reduced sleep results in increased food intake (within certain methodological limitations). Increased intake may eventually lead to weight gain if there is no net compensation over time. These findings suggest a potential relationship with weight gain. However, it is unclear whether the degree of net effect, if any, may be observed over longer periods. None of the included studies that reported food intake went beyond three weeks in duration.

\section{Risk of Bias Analysis}

We found no instances among included studies of apparent selective outcome reporting. This may be due to better journal editing policies of late, since most papers were published 
in the past year. Generally, due to the cross-over nature or planning of the included studies, a lack of an attention placebo was not a common area of concern (only two of seventeen studies). Across all studies, the area of design most commonly found to increase risk of potential bias of outcomes was using a completer's analysis rather man intention to treat. Also, blinding of measurement and other study personnel was not reported or not done in over $60 \%$ of the selected papers. See Figure 6 for summary and itemized author ratings of included studies that were meta-analyzed.

\section{Discussion}

Experimental studies found through our systematic review and meta-analysis do not provide a strong basis to support the presence of a causal relationship between sleep durations and obesity-related factors. However, there are two studies that met our criteria that suggest sleep restriction may contribute to weight gain ${ }^{36,41}$. We also found one ${ }^{43}$ of four studies that indicated total energy expenditure is significantly increased during restricted sleep in adolescents, which might actually be expected to reduce weight unless other compensatory factors, such as a net larger increase in energy intake, are at play. The generalizability of the findings is limited by the small number of studies we found that met our criteria, mostly short intervention periods and the small sample size of included studies. Additionally, there were no studies assessing the effects of long-term manipulation of sleep duration, limiting the power of the overall conclusions. This type of study would be challenging from logistical, ethical and safety considerations.

Our analyses found the benefits of extending sleep modestly effective on weight outcomes overall, but not on other energy balance factors we examined. A possible explanation for this is the difference between lengths of intervention and sleep deficits amongst our included studies. Changes in body weight can be the result of changes in energy stores or changes in hydration and alimentary tract contents ${ }^{46}$. While the effects of sleep interventions on some aspects of energy balance can be observed in 24 hours, it is unclear if tissue-level weight loss is occurring (as opposed to changes in liver glycogen and associated total water loss) in studies that have an intervention duration of less than four weeks.

Bothelius et al. ${ }^{30}$ and Logue et al. ${ }^{28}$ reported improvements in sleep as a result of either cognitive behavioral therapy or a combination of sleep and weight education, and Puhan et al. sought to teach a new breathing skill as a means to decrease sleepiness ${ }^{29}$. It appears that interventions that include education may be helpful in the promotion of reducing sleep problems, and improving sleep quality, and/or sleep duration but with so few RCTs published to date it is an area which needs further research. A limitation of the RCTs we found is that there was not a consistent use of panels of biomarkers of appetite regulating hormones where the intervention was also not confounded by other factors besides sleep duration. There are other quasi-experimental studies (using pre-post designs) examining a variety of appetite-regulating biomarkers in greater numbers, but we found no RCTs other than those we analyzed. Future research synthesis might include comparisons of other interventions (e.g. continuous positive airway pressure, drugs) for their effects on energy balance outcomes. 
The findings concerning energy expenditure demonstrate that a minimum of two days is sufficient in illustrating the effect of sleep manipulation on some metabolic parameters. It is unclear if these changes are enduring in magnitude over time. In our analysis, a majority of studies that restricted sleep found that total energy expenditure and respiratory quotient were not increased with short sleep with the exception of the study on adolescent males ${ }^{43}$. A recent review by Klingenberg et al. ${ }^{47}$ concluded that reduced sleep does not consistently affect energy expenditure in the same magnitude and direction. The majority of the included studies were crossover randomized controlled trials with a maximum sample size of 20 . The studies with the largest samples were simple RCTs and also had a longer intervention period (up to 16 weeks) ${ }^{28-31}$. What remains to be determined is what aspect of sleep (quality, time, wave type, or some combination thereof) is essential to promoting weight loss; it is unclear if this is possible in a segment of the population. Additionally, better understanding of the link between longer waking periods and increased food intake patterns is necessary to discern whether effects are due to more metabolic shifts independent of wake-time activities, or that during chronic periods of sleep restriction, total energy intake is significantly increased without compensation by changes in resting metabolic rate or physical activity. The four RCTs we evaluated that reported the effects of short sleep duration on TEE showed an overall non-significant increase (Figure 4, Panel A). However, only one of these also reported body weight changes ${ }^{35}$, which was not statistically significant (observed for a two week intervention).

An important note about the scope of the present analysis is that we did not examine studies in which specific markers or defined diagnoses of disturbed circadian rhythms were evaluated. We did not include circadian related search terms in our literature search since the ecological reports of reduced sleep time and increased body weights are agnostic of such dynamics, outside of examinations of problems with shift work where these disruptions may be assumed ${ }^{48}$. Our search strategy is sufficient to yield RCTs pertinent to our more focused research question. We aimed to begin building and highlighting the limited evidence base to address epidemiological observations that may or may not have strong causal links. While such circadian indicators may reflect not only altered phase shift or duration-related patterns of sleep, and have been shown to affect some health outcomes that are related to energy balance such as glucose homeostasis ${ }^{15}$, we desired to start with observations of basic phenotypic effects. This does not rule out other potential effects that may be cumulative in some way over a longer period of time.

Further, while there is a larger literature on the use of sleep enhancing substances or devices, e.g. CPAP, exogenous melatonin or prescription drugs, we sought the literature on interventions focused on sleep duration alone since other types of interventions may have differing effects on sleep quality or hormonal regulation of body weight. If recent theories and cohort studies about the association between reduction in total sleep time and increased body weight or adiposity are to be tested, the intervention must not involve such confounding elements. However, it must be acknowledged that evaluations of circadian rhythm-related phenomena are important to include in such considerations both in selection of study participants and timing of study protocol activities. For example, timing of when such hormone samples as insulin and ghrelin are collected may need to be evaluated in individual participants before study protocols begin, rather than using standardized 
collection times based on an assumption that all participants' diurnal cycles are synchronized ${ }^{16}$.

In the past 10 years, there have been studies to examine the role of potentially altered hormone levels due to diurnal disruption as a possible mechanism of increased food intake during sleep restriction. However, a recent review by Chaput and St-Onge ${ }^{49}$ concluded that hormones such as ghrelin and leptin are not the main mechanism contributing to increased food intake during sleep restriction because hormone levels depend on sleep timing and the nutritional state of the participants. They assert that hedonic factors are likely stronger. In the present analysis, all studies testing ghrelin used the assay for total ghrelin rather than the active form. This approach may limit interpretation on the dynamics of this relatively weak meal initiation signal.

Some research has focused on individuals that experience these altered sleep patterns on a daily basis (e.g. shift workers, pilots, caregivers). These conditions may offer opportunities for more insight, There are practical limitations to keeping humans in metabolic chambers tor an extended period of time or to adequately measure outcomes in free-living conditions ${ }^{46}$. Some additional obstacles to this type of well-controlled research are 1) the inability to conduct double blind placebo controlled experiments 2) increasing or reducing sleep in a non-pharmaceutical way and 3) lack of financial reward to appeal to the private sector ${ }^{50}$ for financial support. Examining sleep duration alone may be an oversimplification. Our decision to exclude other parameters was aimed at filling in an important gap in the present state of knowledge. The exclusion of other factors is a limitation to this review and meta-analysis but we aimed to guide future, well-controlled interventional studies over longer periods of time that can build on the present knowledge base.

In conclusion, this systematic review and meta-analysis highlights some initial experimental data suggesting that the manipulation of sleep duration or quality may have significant effects on body composition and other energy balance related factors. The findings of this analysis call for additional research using more standardized methods and measures, as well as evaluations of associated outcomes with sufficient sample sizes and durations to ascertain whether a causal relationship truly exists between sleep duration and body weight regulation. Only then can we attempt to quantify the strength of any relationship.

\section{Acknowledgments}

This project was supported in part by National Institutes of Health grants P30DK056336, K12GM088010, and T32HL072757. We would also like to thank Peng Li, Ph.D. for reviewing the statistical analyses. The content is solely the responsibility of the authors and does not necessarily represent the official views of the National Institutes of Health or any other organization.

\section{Literature Cited}

1. World Health Organization. Obesity and Overweight World Health Organization. 2015. Available at: http://www.who.int/mediacentre/factsheets/fs311/en/

2. National Center for Health Statistics. Health, United States, 2011: With Special Features on Socioeconomic Status and Health. In: U.S. Dept. of Health and Human Services., editor. Hyattsville, MD: 2012. Available at: http://www.cdc.gov/nchs/data/hus/hus11.pdf 
3. Reaven GM. Banting Lecture 1988: Role of Insulin Resistance in Human Disease. Diabetes. 1988; 37:1595-607. [PubMed: 3056758]

4. Vendrell J, Broch M, Vilarrasa N, et al. Resistin, Adiponectin, Ghrelin, Leptin, and Proinflammatory Cytokines: Relationships in Obesity. Obes Res. 2004; 12:962-71. [PubMed: 15229336]

5. National Sleep Foundation. 2013 Sleep in America Poll. In: Foundation NS. , editor. Washington, D.C.: 2013. Available at: http://sleepfoundation.org/sites/default/files/RPT336\%20Summary\%20of \%20Findings\%2002\%2020\%202013.pdf

6. Matricciani L, Olds T, Petkov J. In Search of Lost Sleep: Secular Trends in the Sleep Time of School-Aged Children and Adolescents. Sleep Med Rev. 2012; 16:203-11. [PubMed: 21612957]

7. Keyes KM, Maslowsky J, Hamilton A, Schulenberg J. The Great Sleep Recession: Changes in Sleep Duration among Us Adolescents, 1991-2012. Pediatrics. 2015; 135:460-8. [PubMed: 25687142]

8. Lallukka T, Sares-Jaske L, Kronholm E, et al. Sociodemographic and Socioeconomic Differences in Sleep Duration and Insomnia-Related Symptoms in Finnish Adults. BMC Public Health. 2012; 12:565. [PubMed: 22839359]

9. Whinnery J, Jackson N, Rattanaumpawan P, Grandner MA. Short and Long Sleep Duration Associated with Race/Ethnicity, Sociodemographics, and Socioeconomic Position. Sleep. 2014; 37:601-11. [PubMed: 24587584]

10. Cain N, Gradisar M. Electronic Media Use and Sleep in School-Aged Children and Adolescents: A Review. Sleep Med. 2010; 11:735-42. [PubMed: 20673649]

11. Akerstedt T. Shift Work and Disturbed Sleep/Wakefulness. OccupMed (Lond). 2003; 53:89-94.

12. Taheri S. The Link between Short Sleep Duration and Obesity: We Should Recommend More Sleep to Prevent Obesity. Arch Dis Child. 2006; 91:881-4. [PubMed: 17056861]

13. Patel SR, Blackwell T, Redline S, et al. The Association between Sleep Duration and Obesity in Older Adults. 2008; 32:1825-34.

14. Koren D, Levitt Katz LE, Brar PC, Gallagher PR, Berkowitz RI, Brooks LJ. Sleep Architecture and Glucose and Insulin Homeostasis in Obese Adolescents. Diabetes Care. 2011; 34:2442-7. [PubMed: 21933909]

15. Spiegel K, Tasali E, Leproult R, Van Cauter E. Effects of Poor and Short Sleep on Glucose Metabolism and Obesity Risk. Nat Rev Endocrinol. 2009; 5:253-61. [PubMed: 19444258]

16. Taheri S, Lin L, Austin D, Young T, Mignot E. Short Sleep Duration Is Associated with Reduced Leptin, Elevated Ghrelin, and Increased Body Mass Index. PLoS Med. 2004; 1:e62. [PubMed: 15602591]

17. Van Cauter E, Holmback U, Knutson K, et al. Impact of Sleep and Sleep Loss on Neuroendocrine and Metabolic Function. Horm Res. 2007; 67(Suppl 1):2-9. [PubMed: 17308390]

18. Brondel L, Romer MA, Nougues PM, Touyarou P, Davenne D. Acute Partial Sleep Deprivation Increases Food Intake in Healthy Men. Am J Clin Nutr. 2010; 91:1550-9. [PubMed: 20357041]

19. St-Onge MP. The Role of Sleep Duration in the Regulation of Energy Balance: Effects on Energy Intakes and Expenditure. J Clin Sleep Med. 2013; 9:73-80. [PubMed: 23319909]

20. Beebe UW, Simon S, Summer S, Hemmer S, Strotman D, Dolan LM. Dietary Intake Following Experimentally Restricted Sleep in Adolescents. Sleep. 2013; 36:827-34. [PubMed: 23729925]

21. Simon SL, Field J, Miller LE, DiFrancesco M, Beebe DW. Sweet/Dessert Foods Are More Appealing to Adolescents after Sleep Restriction. PLoS One. 2015; 10:e0115434. [PubMed: 25706861]

22. Cappuccio FP, Taggart FM, Kandala NB, et al. Meta-Analysis of Short Sleep Duration and Obesity in Children and Adults. Sleep. 2008; 31:619-26. [PubMed: 18517032]

23. Chen X, Beydoun MA, Wang Y. Is Sleep Duration Associated with Childhood Obesity? A Systematic Review and Meta-Analysis. Obesity (Silver Spring). 2008; 16:265-74. [PubMed: 18239632]

24. Patel SR, Hu FB. Short Sleep Duration and Weight Gain: A Systematic Review. Obesity (Silver Spring). 2008; 16:643-53. [PubMed: 18239586] 
25. Thind H, Davies SL, Lewis T, Pekmezi D, Evans R, Baskin ML. Does Short Sleep Lead to Obesity among Children and Adolescents?: Current Understanding and Implications. American Journal of Lifestyle Medicine. 2014

26. Centre CTNC. Review Manager (Revman) [Computer Program] Version 5.1.6. The Cochrane Collaboration. 2011

27. J.P.T. H, S. G. , editor. The Cochrane Collaboration. The Cochrane Collaboration; 2011. Cochrane Handbook for Systematic Reviews of Interventions Version 5.1.0 [Updated March 2011].

28. Logue EE, Bourguet CC, Palmieri PA, et al. The Better Weight-Better Sleep Study: A Pilot Intervention in Primary Care. Am J Health Behav. 2012; 36:319-34. [PubMed: 22370434]

29. Puhan MA, Suarez A, Lo Cascio C, Zahn A, Heitz M, Braendli O. Didgeridoo Playing as Alternative Treatment for Obstructive Sleep Apnoea Syndrome: Randomised Controlled Trial. BMJ. 2006; 332:266-70. [PubMed: 16377643]

30. Bothelius K, Kyhle K, Espie CA, Broman JE. Manual- Guided Cognitive-Behavioural Therapy for Insomnia Delivered by Ordinary Primary Care Personnel in General Medical Practice: A Randomized Controlled Effectiveness Trial. Journal of Sleep Research. 2013; 22:688-96. [PubMed: 23859625]

31. Calvin AD, Carter RE, Adachi T, et al. Effects of Experimental Sleep Restriction on Caloric Intake and Activity Energy Expenditure. Chest. 2013; 144:79-86. [PubMed: 23392199]

32. Chapman CD, Nilsson EK, Nilsson VC, et al. Acute Sleep Deprivation Increases Food Purchasing in Men. Obesity. 2013; 21:E555-E60. [PubMed: 23908148]

33. Hogenkamp PS, Nilsson E, Nilsson VC, et al. Acute Sleep Deprivation Increases Portion Size and Affects Food Choice in Young Men. Psychoneuroendocrinology. 2013; 38:1668-74. [PubMed: 23428257]

34. Hursel R, Gonnissen HKJ, Rutters F, Martens EAP, Westerterp-Plantenga MS. Disadvantageous Shift in Energy Balance Is Primarily Expressed in High-Quality Sleepers after a Decline in Quality Sleep Because of Disturbance. American Journal of Clinical Nutrition. 2013; 98:367-73. [PubMed: 23803894]

35. Nedeltcheva AV, Kilkus JM, Imperial J, Kasza K, Schoeller DA, Penev PD. Sleep Curtailment Is Accompanied by Increased Intake of Calories from Snacks. Am J Clin Nutr. 2009; 89:126-33. [PubMed: 19056602]

36. O'Keeffe M, Roberts AL, Kelleman M, RoyChoudhury A, St-Onge M-P. No Effects of Short-Term Sleep Restriction, in a Controlled Feeding Setting, on Lipid Profiles in Normal-Weight Adults. Journal of Sleep Research. 2013; 22:717-20. [PubMed: 23682639]

37. Shechter A, Rising R, Albu JB, St-Onge MP. Experimental Sleep Curtailment Causes WakeDependent Increases in 24-H Energy Expenditure as Measured by Whole-Room Indirect Calorimetry. Am J Clin Nutr. 2013; 98:1433-9. [PubMed: 24088722]

38. Simpson NS, Banks S, Dinges DF. Sleep Restriction Is Associated with Increased Morning Plasma Leptin Concentrations, Especially in Women. Biol Res Nurs. 2010; 12:47-53. [PubMed: 20453022]

39. Dzaja A, Dalai MA, Himmerich H, Uhr M, Pollmacher T, Schuld A. Sleep Enhances Nocturnal Plasma Ghrelin Levels in Healthy Subjects. Am J Physiol Endocrinol Metab. 2004; 286:E963-7. [PubMed: 14871884]

40. Spiegel K, Tasali E, Penev P, Van Cauter E. Brief Communication: Sleep Curtailment in Healthy Young Men Is Associated with Decreased Leptin Levels, Elevated Ghrelin Levels, and Increased Hunger and Appetite. Ann Intern Med. 2004; 141:846-50. [PubMed: 15583226]

41. Spaeth AM, Dinges DF, Goel N. Effects of Experimental Sleep Restriction on Weight Gain, Caloric Intake, and Meal Timing in Healthy Adults. Sleep. 2013; 36:981-90. [PubMed: 23814334]

42. Hart CN, Carskadon MA, Considine RV, et al. Changes in Children's Sleep Duration on Food Intake, Weight, and Leptin. Pediatrics. 2013; 132:e1473-80. [PubMed: 24190680]

43. Klingenberg L, Chaput JP, Holmback U, Jennum P, Astrup A, Sjodin A. Sleep Restriction Is Not Associated with a Positive Energy Balance in Adolescent Boys. Am J Clin Nutr. 2012; 96:240-8. [PubMed: 22760574] 
44. Schmid SM, Hallschmid M, Jauch-Chara K, et al. Short-Term Sleep Loss Decreases Physical Activity under Free-Living Conditions but Does Not Increase Food Intake under Time-Deprived Laboratory Conditions in Healthy Men. Am J Clin Nutr. 2009; 90:1476-82. [PubMed: 19846546]

45. St-Onge MP, Roberts AL, Chen J, et al. Short Sleep Duration Increases Energy Intakes but Does Not Change Energy Expenditure in Normal-Weight Individuals. Am J Clin Nutr. 2011; 94:410-6. [PubMed: 21715510]

46. Hall KD, Heymsfield SB, Kemnitz JW, Klein S, Schoeller DA, Speakman JR. Energy Balance and Its Components: Implications for Body Weight Regulation. Am J Clin Nutr. 2012; 95:989-94. [PubMed: 22434603]

47. Klingenberg L, Sjodin A, Holmback U, Astrup A, Chaput JP. Short Sleep Duration and Its Association with Energy Metabolism. Obes Rev. 2012; 13:565-77. [PubMed: 22440089]

48. Sack RL, Auckley D, Auger RR, et al. Circadian Rhythm Sleep Disorders: Part I, Basic Principles, Shift Work and Jet Lag Disorders. An American Academy of Sleep Medicine Review. Sleep. 2007; 30:1460-83. [PubMed: 18041480]

49. Chaput JP, St-Onge MP. Increased Food Intake by Insufficient Sleep in Humans: Are We Jumping the Gun on the Hormonal Explanation? Front Endocrinol (Lausanne). 2014; 5:116. [PubMed: 25076940]

50. Cizza G, Skarulis M, Mignot E. A Link between Short Sleep and Obesity: Building the Evidence for Causation. Sleep. 2005; 28:1217-20. [PubMed: 16295203]

51. Hursel R, Rutters F, Gonnissen HK, Martens EA, Westerterp-Plantenga MS. Effects of Sleep Fragmentation in Healthy Men on Energy Expenditure, Substrate Oxidation, Physical Activity, and Exhaustion Measured over $48 \mathrm{H}$ in a Respiratory Chamber. Am J Clin Nutr. 2011; 94:804-8. [PubMed: 21795436]

52. Klingenberg L, Chaput JP, Holmbadck U, et al. Acute Sleep Restriction Reduces Insulin Sensitivity in Adolescent Boys. Sleep. 2013; 36:1085-90. [PubMed: 23814346]

53. Markwald RR, Melanson EL, Smith MR, et al. Impact of Insufficient Sleep on Total Daily Energy Expenditure, Food Intake, and Weight Gain. Proc Natl Acad Sci USA. 2013; 110:5695-700. [PubMed: 23479616]

\section{Abbreviations}

$\begin{array}{ll}\text { BMI } & \text { body mass index } \\ \text { CPAP } & \text { continuous positive airway pressure } \\ \text { d } & \text { days } \\ \text { h } & \text { hour } \\ \text { RCT } & \text { randomized controlled trial } \\ \text { RQ } & \text { respiratory quotient } \\ \text { TEE } & \text { total energy expenditure }\end{array}$




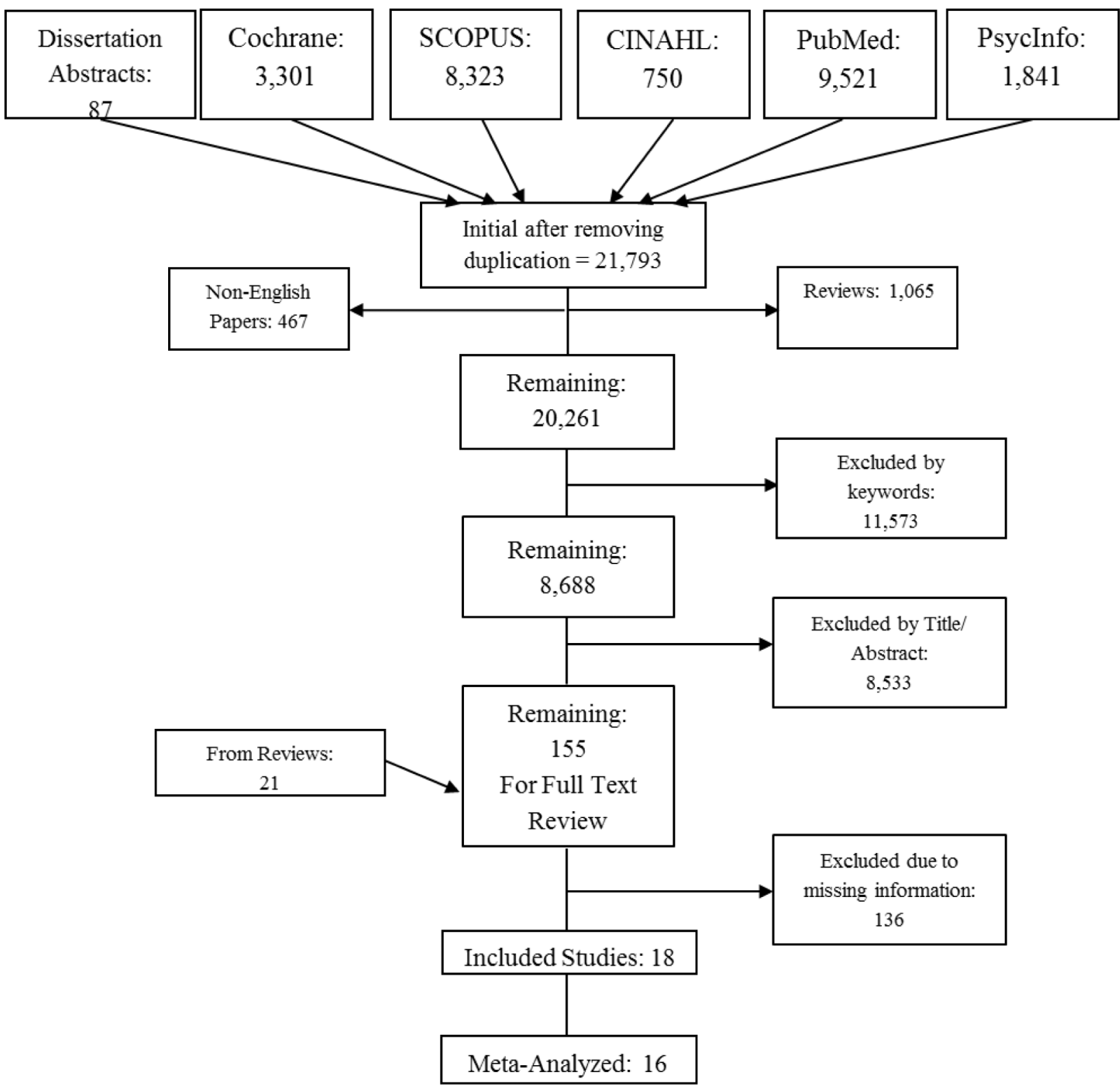

Figure 1. PRISMA diagram and study search and selection process 


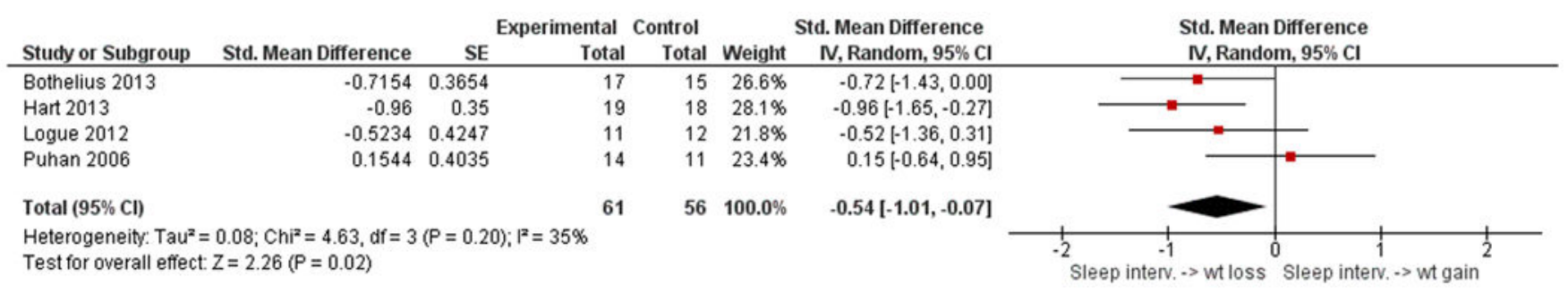

Figure 2. Comparison of sleep improvement intervention studies and the effect on body weight compared to control 


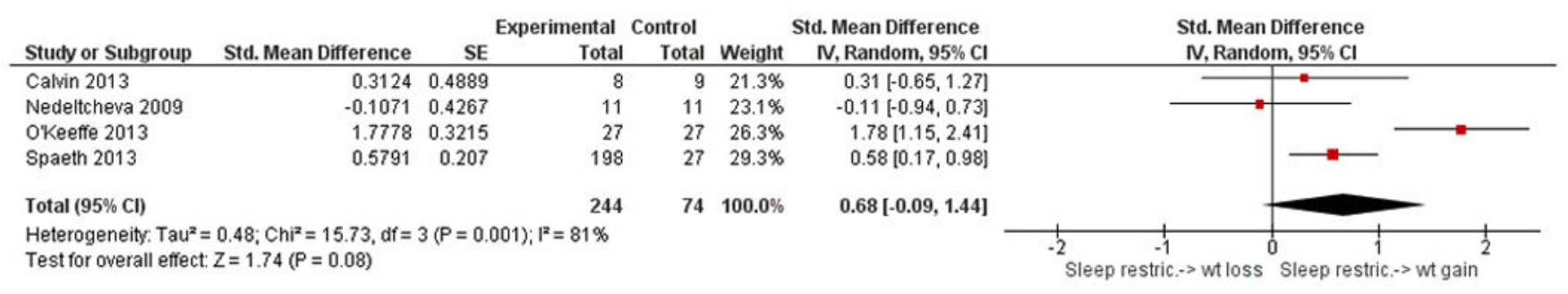

Figure 3. Comparison of studies of sleep restriction and the effect on body weight compared to control 


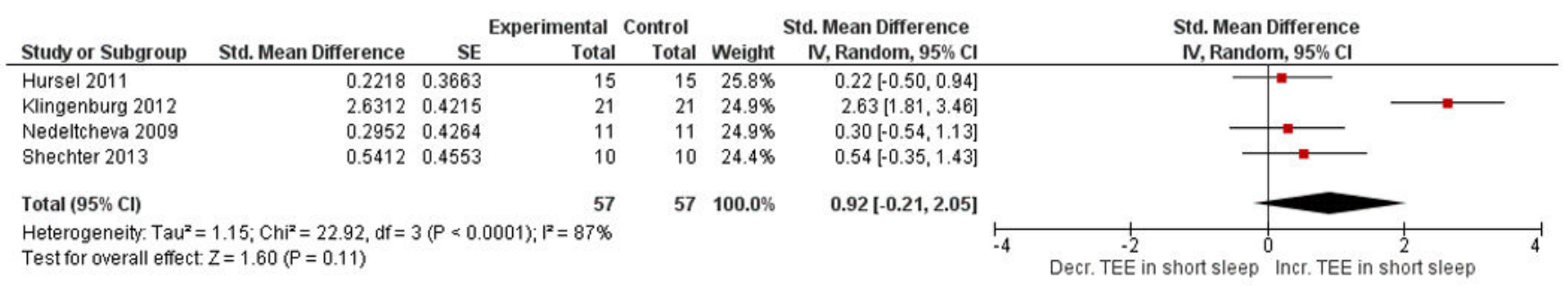

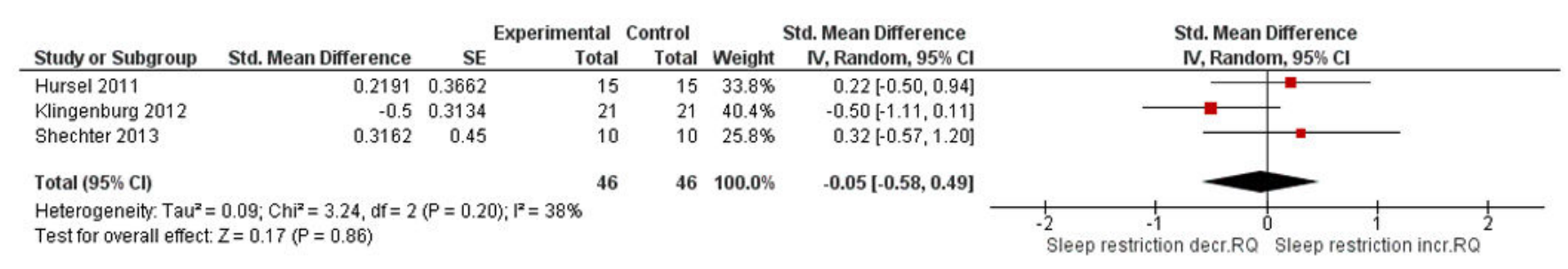

Figure 4. Comparison of studies of sleep restriction on metabolic chamber measures of total energy expenditure (TEE, Panel A) or respiratory quotient (RQ, Panel B) 


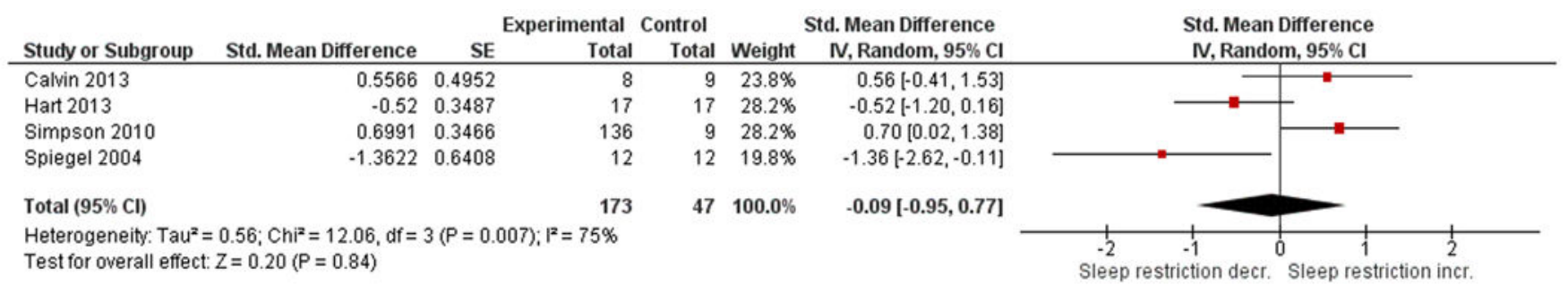

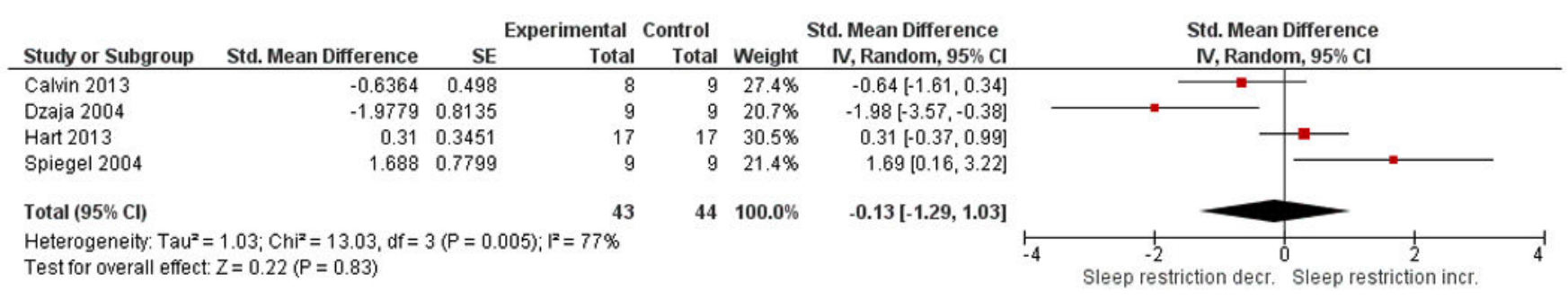

Figure 5. Comparison of studies of sleep restriction on leptin (Panel 5A) and ghrelin (Panel 5B) 


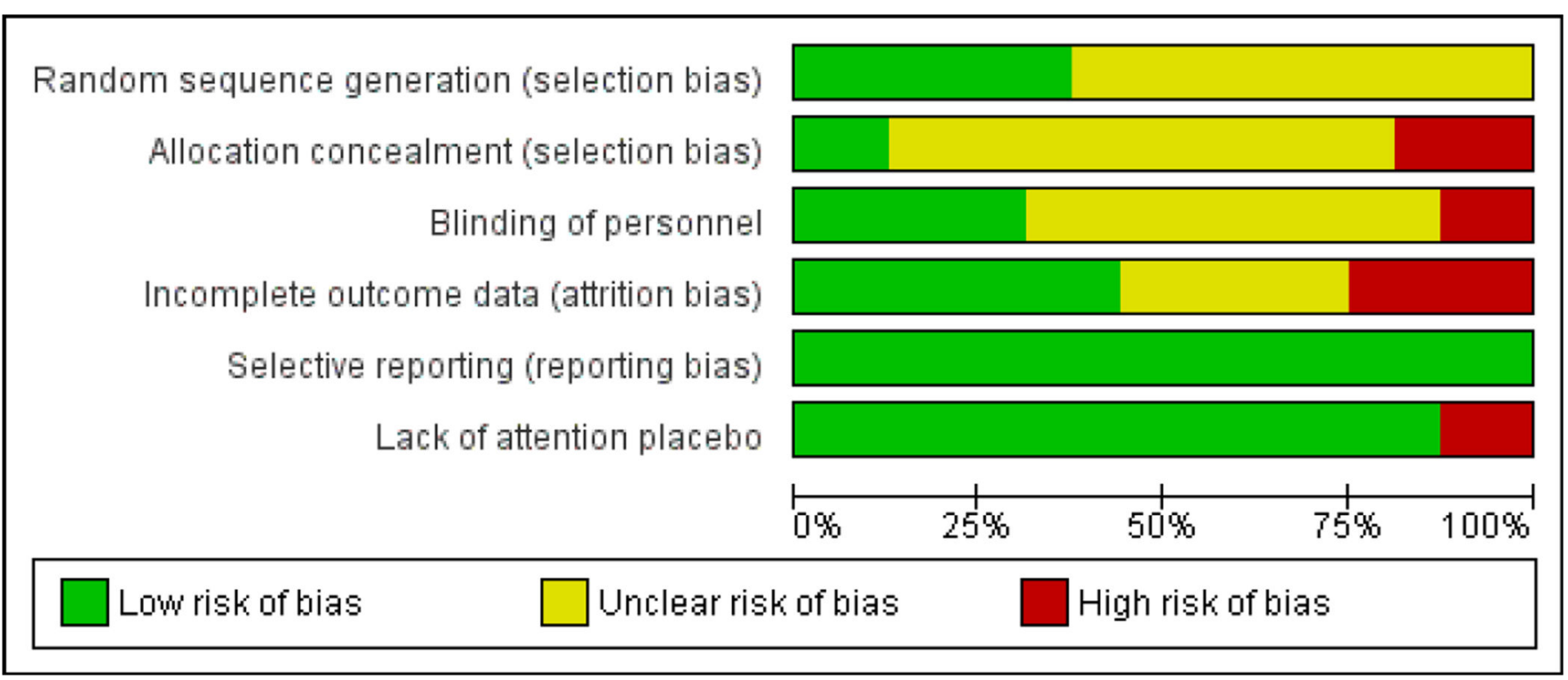




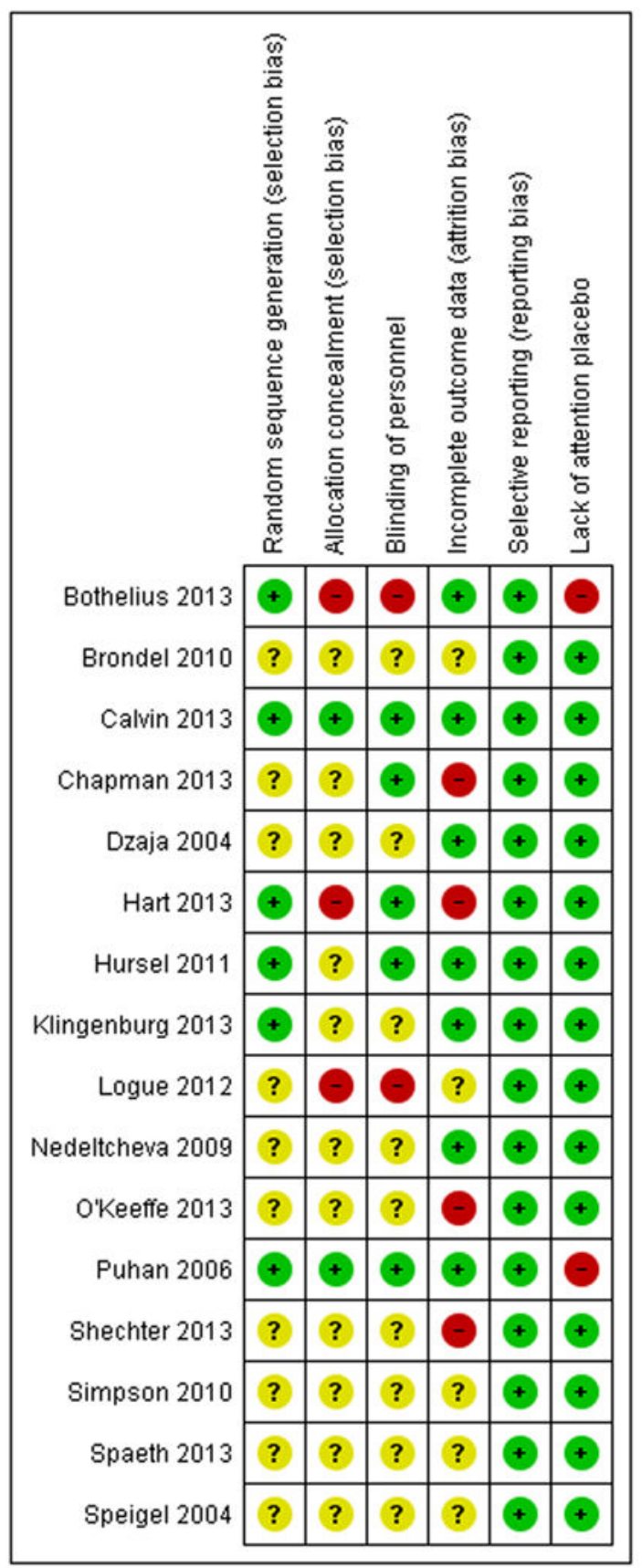

Figure 6. Authors' judgments of risk of bias assessment per paper and overall summary 


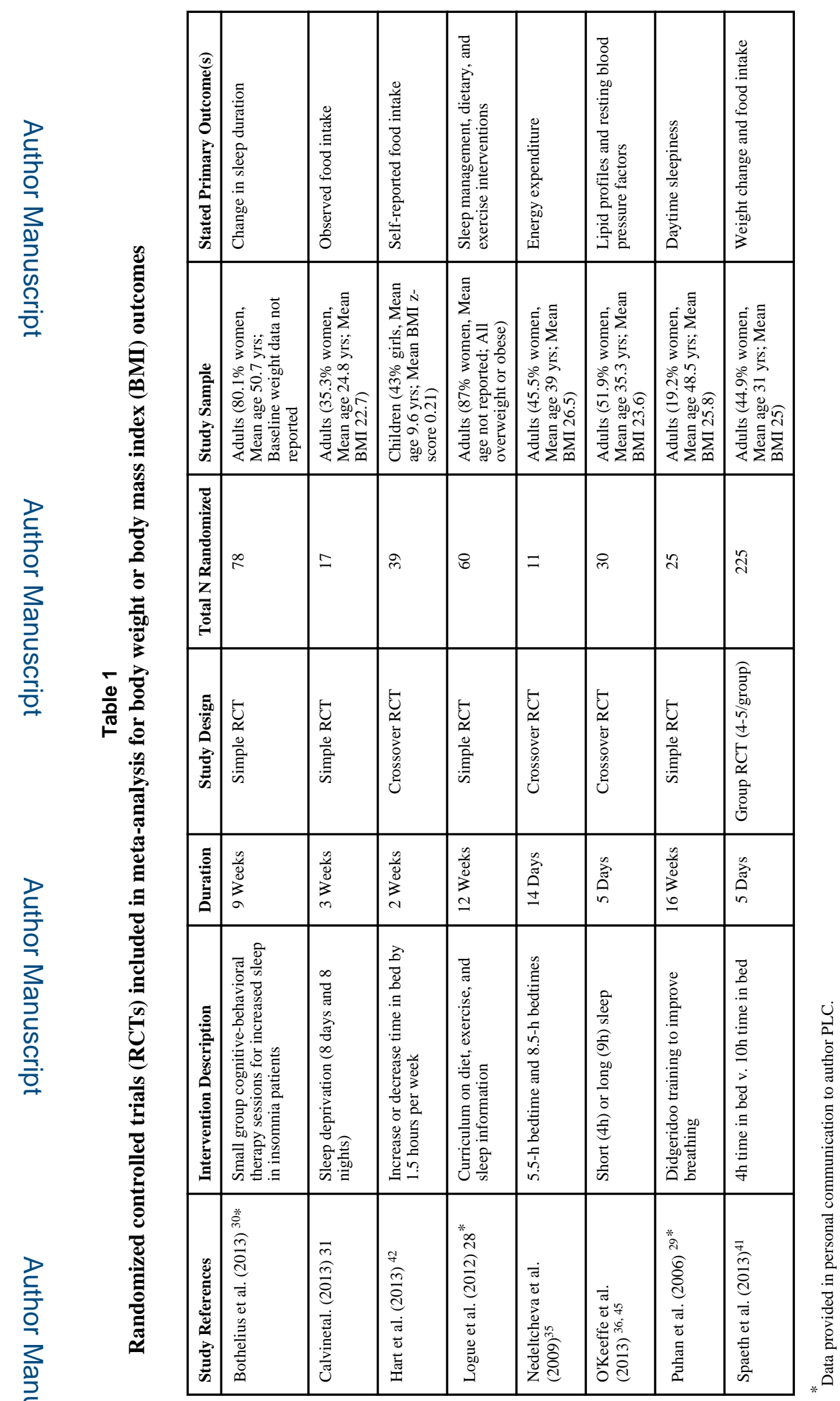

Obes Rev. Author manuscript; available in PMC 2016 September 01. 


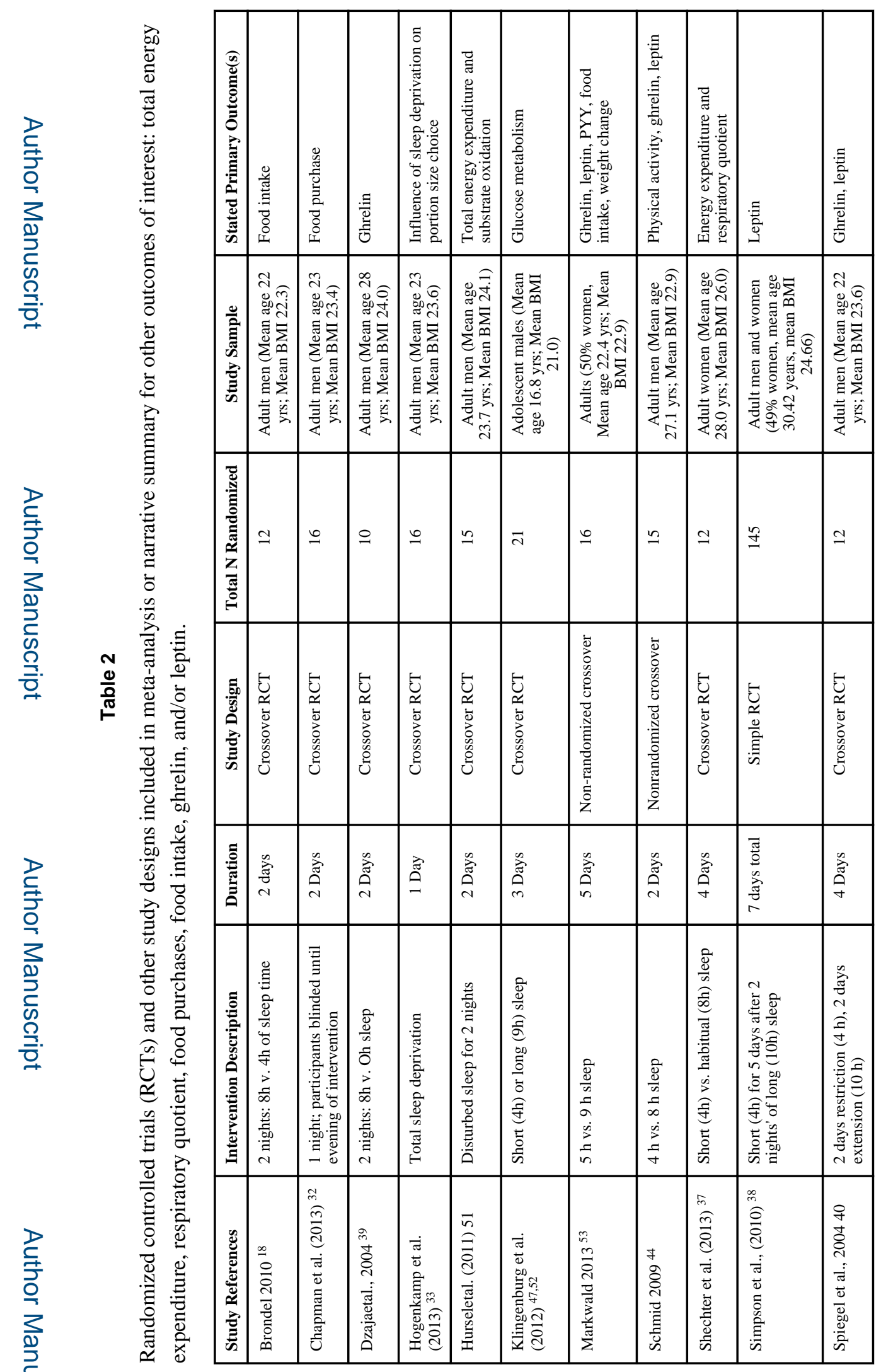

Obes Rev. Author manuscript; available in PMC 2016 September 01. 